\title{
No difference in outcome between children and adolescents transplanted for acute lymphoblastic leukemia in second remission
}

\author{
Giorgio Dini, ${ }^{1}$ Marco Zecca, ${ }^{2}$ Adriana Balduzzi, ${ }^{3}$ Chiara Messina, ${ }^{4}$ Riccardo Masetti, ${ }^{5}$ Franca Fagioli, ${ }^{6}$ Claudio Favre,${ }^{7}$ \\ Marco Rabusin, ${ }^{8}$ Fulvio Porta, ${ }^{9}$ Erika Biral, ${ }^{1}$ Mimmo Ripaldi, ${ }^{10}$ Anna Paola lori, ${ }^{11}$ Carla Rognoni, ${ }^{12}$ Arcangelo Prete, ${ }^{5}$ and \\ Franco Locatelli, ${ }^{13,14}$ on behalf of the Associazione Italiana Ematologia ed Oncologia Pediatrica-Hematopoietic Stem Cell \\ Transplantation (AIEOP-HSCT) Group \\ 1Dipartimento Ematologia e Oncologia Pediatrica, Istituto di Ricovero e Cura a Carattere Scientifico (IRCCS) G. Gaslini, Genova, Italy; ${ }^{2}$ Oncoematologia \\ Pediatrica, Fondazione IRCCS Policlinico “San Matteo," Pavia, Italy; ${ }^{3}$ Clinica Pediatrica, Università di Milano Bicocca, Ospedale San Gerardo, Monza, Italy; \\ ${ }^{4}$ Oncoematologia Pediatrica, Dipartimento di Pediatria, Università di Padova, Padova, Italy; ${ }^{5}$ Clinica Pediatrica, Università di Bologna, Policlinico Sant'Orsola, \\ Bologna, Italy; ${ }^{6}$ Ospedale Regina Margherita, Università di Torino, Torino, Italy; ${ }^{7}$ Clinica Pediatrica, Università di Pisa, Pisa, Italy; ${ }^{8}$ Oncoematologia Pediatrica, \\ IRCCS Burlo Garofolo, Trieste, Italy; ${ }^{9}$ Pediatric Oncohaematology and BMT Unit, Children's Hospital, Brescia, Italy; ${ }^{10}$ Oncoematologia Pediatrica, Ospedale \\ Pausilipon, Napoli, Italy; ${ }^{11}$ Cattedra di Ematologia, Università La Sapienza, Roma, Italy; ${ }^{12}$ Laboratorio di Informatica Biomedica, Dipartimento di Informatica e \\ Sistemistica, Università di Pavia, Pavia, Italy; ${ }^{13}$ Dipartimento di Oncoematologia Pediatrica, IRCCS Ospedale Bambino Gesù, Roma, Italy; and ${ }^{14}$ Università di \\ Pavia, Pavia, Italy
}

Acute lymphoblastic leukemia (ALL) in second complete remission is one of the most common indications for allogeneic hematopoietic stem cell transplantation (HSCT) in pediatric patients. We compared the outcome after HCST of adolescents, aged 14 to 18 years, with that of children (ie, patients $<14$ years of age). Enrolled in the study were 395 patients given the allograft between January 1990 and December 2007; both children (334) and adolescents (61) were transplanted in the same pediatric institutions. All patients received a myeloablative regimen that included total body irradiation in the majority of them. The donor was an HLAidentical sibling for 199 patients and an unrelated volunteer in the remaining 196 patients. Children and adolescents had a comparable cumulative incidence of transplantation-related mortality, disease recurrence, and of both acute and chronic graft-versus-host disease. The 10-year probability of overall survival and event-free survival for the whole cohort of patients were $57 \%$ (95\% confidence interval, $52 \%-62 \%$ ) and $54 \%$ (95\% confidence interval, $49 \%-59 \%$ ), respectively, with no difference between children and adolescents. This study documents that adolescents with ALL in second complete remission given HSCT in pediatric centers have an outcome that does not differ from that of patients younger than 14 years of age. (Blood. 2011;118(25):6683-6690)

\section{Introduction}

Nowadays, intensive and risk-adapted chemotherapy protocols cure more than $80 \%$ of children with acute lymphoblastic leukemia (ALL). ${ }^{1,2}$ Disease recurrence remains the leading cause of treatment failure in childhood ALL. ${ }^{3}$ Among ALL patients developing recurrence, a substantial proportion achieves a second complete remission (CR), although final outcome remains still unsatisfactory. In this respect, survival of patients experiencing relapse can be predicted by site of relapse, duration of first complete remission, and immunophenotype of ALL. Bone marrow (BM) relapse and early relapse (ie, occurring within 6 months from treatment discontinuation), as well as T-lineage ALL, have worse prognosis than isolated extramedullary or late relapse (ie, those occurring more than 6 months from treatment discontinuation). ${ }^{3}$ Allogeneic hematopoietic stem cell transplantation (HSCT) is largely used in the post-remission treatment of children and adolescents with ALL in second CR (CR2). ${ }^{3}$ In the past, analysis of factors influencing the outcome of patients transplanted from an unrelated volunteer showed that adolescents had a poorer outcome compared with children. ${ }^{4,5}$ With the aim of verifying whether this difference is confirmed in another cohort, we compared the outcome of HCST in

Submitted May 13, 2011; accepted September 13, 2011. Prepublished online as Blood First Edition paper, October 18, 2011; DOI 10.1182/blood-2011-05354233.

The online version of the article contains a data supplement. adolescents, defined as 14 years or older, with that of children younger than 14 years, with relapsed ALL who were reported to the Associazione Italiana Ematologia ed Oncologia Pediatrica (AIEOP).

\section{Patients and methods}

\section{Patients}

Enrolled in the study were 395 patients (256 males and 139 females) younger than 18 years, with ALL in CR2 after either isolated or combined extramedullary/BM relapse, who have received a first allogeneic HSCT from either a matched family donor (MFD) or an unrelated donor (UD) in one of the 18 AIEOP centers performing allogeneic HSCT. Patients were given the allograft between January 1, 1990, and December 31, 2007, and were reported to the AIEOP-HSCT Registry. Data concerning patient and disease characteristics, as well as transplantation outcome, were collected by a standardized questionnaire for each patient enrolled.

Histocompatibility for all donor-recipient pairs was determined by serology or low-resolution molecular typing for HLA-A and -B antigens and high-resolution allelic technique for DRB1. All MFD pairs were A, B, DRB1 matched. Since 1998, high-resolution molecular typing also has been marked "advertisement" in accordance with 18 USC section 1734.

(c) 2011 by The American Society of Hematology 
performed for HLA class I loci in most of patients transplanted from an UD, and we defined this characterization as high-resolution typing. Pairs matched for HLA-A, -B, and -DRB1 were defined as 6/6-loci matched, whereas pairs in which there was incompatibility for a single HLA locus were defined as 5/6-loci matched. All UD pairs were either 5- or 6-loci matched.

At time of transplantation, 334 patients were younger than 14 years, whereas the remaining 61 patients where 14 years or older, henceforth defined as adolescents.

In both groups, $>90 \%$ of the transplants were performed using BM stem cells. Preparative regimens varied, mainly depending on patient age and center choice. Nevertheless, all patients received a myeloablative conditioning regimen that included total body irradiation in $89 \%$ of the cases, most often associated with thiotepa and cyclophosphamide. ${ }^{6}$

Graft-versus-host disease (GVHD) prophylaxis consisted of cyclosporine (CsA) alone for $87 \%$ of MFD recipients and of a combination of CsA, short-term methotrexate and rabbit anti-thymocyte globulin (2.5-3.75 $\mathrm{mg} / \mathrm{kg} / \mathrm{day}$ from day -4 to day -2 ) for $77 \%$ of UD recipients. The children and adolescent groups were comparable for all the variables analyzed, except for the median white blood cell count at diagnosis that was higher in the former group (see Table 1 for details).

Acute and chronic GVHD (aGvHD, cGvHD) were diagnosed and graded according to the Seattle criteria. ${ }^{7,8}$ Patients surviving more than 14 and 100 days after transplantation were evaluated for aGvHD and cGvHD occurrence, respectively. Whenever clinically indicated and feasible, tissue biopsy samples were obtained to confirm the diagnosis of GVHD. ${ }^{9,10}$

Myeloid engraftment was defined as the first of 3 consecutive days when neutrophil count was $>0.5 \times 10^{9} / \mathrm{L}$, and platelet engraftment was defined as the first of 7 consecutive days with an unsupported platelet count $>50 \times 10^{9} / \mathrm{L}$

Children were stratified into 4 risk groups (S1, S2, S3, and S4), according to the Berlin-Frankfurt-Munster (BFM) classification of ALL relapses, based on immune-phenotype, site of relapse, and time elapsed from diagnosis to relapse. ${ }^{11}$ On this basis, $17 \%$ of the children and $21 \%$ of the adolescents were included in the S4 group consisting of patients experiencing marrow relapse, of either T-lineage ALL, irrespective of the time interval between diagnosis and recurrence, or of B-lineage ALL, if relapse occurred within the first 18 months after diagnosis. Eleven percent of the children and 3\% of the adolescents were included in the S3 group consisting of isolated marrow relapses of B-lineage ALL occurring 18 months after diagnosis and within 6 months after treatment discontinuation. Forty-two percent of the children and $43 \%$ of the adolescent were included in the $\mathrm{S} 2$ groups consisting of isolated or combined marrow relapse of B-lineage ALL occurring more than 6 months after treatment discontinuation, and combined marrow relapses of B-lineage ALL occurring later than 18 months after diagnosis and within 6 months after treatment discontinuation. Three percent of children and none of the adolescents were included in the S1 group, the group associated with the best prognosis and comprises patients with isolated extramedullary relapses occurring later than 6 months after treatment discontinuation. One hundred ten patients could not be included in any of the BFM class of risk because of lack of the relevant data needed for stratification. Details on patient stratification are presented in Table 1 .

\section{Statistical analysis}

Patients' data were collected using patient-oriented forms, filled in by a physician in charge at each center, and sent to the AIEOP Operation Office in Bologna. There the data were stored in an electronic database (AIEOPHSCT Registry) and analyzed for quality control and statistical analysis by Venus, a facilities-integrated software system running on an IBM mainframe at the Italian Inter-University Computing Center.

The primary aim of this study was to evaluate the impact of patient age on the outcome of HSCT in ALL in CR2. We also evaluated the impact on outcome of the following patient-, donor-, and transplantation-related factors: recipient sex, interval between diagnosis and HSCT, interval between relapse and HSCT, BFM risk class at relapse, donor age, donor-recipient sex mismatch, human cytomegalovirus serology for both donor and recipient, conditioning regimen (total body irradiation vs chemotherapy), year of transplantation, number of infused BM-nucleated cells, and occurrence of aGvHD and cGvHD.

Quantitative variables are reported as median and range, whereas categorical variables are expressed as absolute number and percentage. Demographic and clinical characteristics of the 2 groups of patients were compared using the $\chi^{2}$ test or Fisher exact test for categorical variables (eg, sex, disease, conditioning regimen, HLA matching), whereas the Mann-Whitney rank sum test and the student $t$ test were used for continuous variables (eg, age, time interval between diagnosis and HSCT, marrow cell dose), as appropriate.

Overall survival (OS) was defined as the probability of survival, regardless of disease status, at any time point; surviving patients were censored at last follow-up, whereas only death was considered an event. Event-free survival (EFS) was defined as the probability of being alive and disease-free at any time point; both death and relapse were considered events, whereas patients who were alive and disease free were censored at last follow-up. Relapse rate (RR) was defined as the probability of having a relapse at time $t$, death in remission being considered the competing event. On the contrary, treatment-related mortality (TRM) was defined as the probability of dying without previous occurrence of relapse, which was considered to be the competing event. Acute and cGVHD were defined as the probabilities to develop either grade II-IV or grade III-IV aGvHD or cGvHD, considering both graft failure and TRM as competing events. ${ }^{12} \mathrm{OS}$, EFS, RR, TRM, aGvHD, and cGvHD were estimated from the date of transplantation to the date of the statistical analysis or to the date of an adverse event. All results are expressed as 10-year probability or 10-year cumulative incidence (percentage) and $95 \%$ confidence interval $(95 \% \mathrm{CI}){ }^{13}$

Probabilities of OS and EFS were calculated according to the KaplanMeier method. ${ }^{14}$ RR, TRM, aGvHD, and cGvHD were calculated as cumulative incidence curves to adjust the estimates for competing risks..$^{15,16}$ The significance of differences between EFS curves was estimated by the log-rank test (Mantel-Cox), whereas the Gray test was used to assess, in univariate analyses, differences between the cumulative incidences of RR, TRM, aGvHD, and cGvHD. ${ }^{17}$

All variables with a $P$ value $<0.1$ in univariate analysis were included in a multivariate analysis of EFS, performed using the Cox proportional hazard regression model. ${ }^{18}$ The proportional subdistribution hazard regression model was used to perform multivariate analyses of RR and TRM. ${ }^{19}$

Statistical analysis was performed using the R 2.5.0 software package (http://www.R-project.org). ${ }^{20,21}$ Data were analyzed as of May 15, 2010.

\section{Results}

The median observation time for surviving patients, considering the whole study population, was 8 years (range, $2-20$ years); it was 8.7 years for children and 7.0 years for adolescents $(P=$ N.S. $)$.

\section{Engraftment and GVHD}

Three patients (1\%) died before engraftment; the remaining 392 patients engrafted. No significant differences in myeloid and platelet recovery were observed between the 2 groups (data not shown).

The 100-day cumulative incidence (Figure 1) of grade II-IV aGvHD in children or adolescents was 54\% (95\% CI, 49\%-60\%) and $49 \%(95 \% \mathrm{CI}, 37 \%-66 \%$; $P=$ N.S.), whereas the 100 -day cumulative incidence of grade III-IV aGvHD was $19 \%(95 \% \mathrm{CI}$, $15 \%-24 \%$ ) and $14 \%$ (95\% CI, 7\%-28\%), respectively ( $P=$ N.S.).

One hundred and one (34\%) of the 300 patients at risk (ie, surviving at least 100 days after HSCT) developed cGvHD that was limited in 63 cases $(21 \%)$ and extensive in the remaining $38(13 \%)$. The cumulative incidence of cGvHD was $32 \%$ (95\% CI, $27 \%-38 \%$ ) for children and $43 \%$ (95\% CI, 31\%-60\%) for adolescents $(P=$ N.S. $)$, respectively. In 4 cases, cGvHD had de novo 
Table 1. Clinical characteristics of the 395 patients enrolled in the study and comparison between children and adolescents

\begin{tabular}{|c|c|c|c|c|c|}
\hline \multirow[b]{2}{*}{ Characteristic } & \multicolumn{2}{|c|}{ Children } & \multicolumn{2}{|c|}{ Adolescents } & \multirow[b]{2}{*}{$P$} \\
\hline & $\mathbf{n}$ & (\%) & $\mathbf{n}$ & $(\%)$ & \\
\hline No. of patients & 334 & $(100)$ & 61 & (100) & \\
\hline \multicolumn{6}{|l|}{ Sex } \\
\hline Male & 212 & (63) & 44 & (72) & N.S. \\
\hline Female & 122 & (37) & 17 & (28) & \\
\hline Median age at diagnosis, y (range) & 4.9 & $(1.1-13)$ & 13.3 & $(5.6-16.9)$ & $<.001$ \\
\hline Median WBC at diagnosis, $\times 10^{9} / \mathrm{L}$ (range) & 14.5 & $(0.1-430)$ & 8.1 & $(0.3-311)$ & 0.01 \\
\hline \multicolumn{6}{|l|}{ Immunophenotype } \\
\hline B-lineage ALL & 215 & (64) & 35 & (57) & .08 \\
\hline T-lineage ALL & 26 & (8) & 10 & (16) & \\
\hline Missing/unknown & 93 & (28) & 16 & (26) & \\
\hline \multicolumn{6}{|l|}{ Cytogenetic abnormalities } \\
\hline $\mathrm{t}(9 ; 22)$ & 12 & (4) & 3 & (5) & N.S. \\
\hline \multicolumn{6}{|l|}{ Frontline chemotherapy protocol } \\
\hline AIEOP LAL 82 & 6 & (2) & 0 & (0) & N.S. \\
\hline AIEOP LAL 87 & 24 & (7) & 5 & (8) & \\
\hline AIEOP LAL 88 & 20 & (6) & 2 & (3) & \\
\hline AIEOP LAL 91 & 71 & (21) & 11 & (18) & \\
\hline AIEOP LAL 95 & 116 & (34) & 17 & (28) & \\
\hline AIEOP LAL 2000 & 87 & (27) & 21 & (35) & \\
\hline Other & 10 & (3) & 5 & (8) & \\
\hline Median interval between diagnosis and first relapse, $\mathrm{mo}$ (range) & 29 & $(2-121)$ & 33 & $(3-102)$ & N.S. \\
\hline \multicolumn{6}{|l|}{ Relapse timing } \\
\hline Very early relapse $<18 \mathrm{mo}$ from diagnosis & 69 & (21) & 13 & (21) & N.S. \\
\hline Early relapse $18-30$ mo from diagnosis & 94 & (28) & 13 & (21) & \\
\hline Late relapse $>30$ mo from diagnosis & 159 & (48) & 32 & (53) & \\
\hline Missing/unknown & 12 & (3) & 3 & (5) & \\
\hline \multicolumn{6}{|l|}{ Site of first relapse } \\
\hline BM & 232 & (69) & 40 & (65) & N.S. \\
\hline Combined BM + extramedullary & 40 & (12) & 9 & (15) & \\
\hline Isolated extramedullary & 40 & (12) & 6 & (10) & \\
\hline Missing/unknown & 22 & (7) & 6 & (10) & \\
\hline \multicolumn{6}{|l|}{ BFM class at relapse } \\
\hline S1 & 9 & (3) & 0 & (0) & N.S. \\
\hline S2 & 141 & (42) & 26 & (43) & \\
\hline S3 & 38 & (11) & 2 & (3) & \\
\hline S4 & 56 & (17) & 13 & (21) & \\
\hline Missing/unknown & 90 & (27) & 20 & (33) & \\
\hline Median age at HSCT, y (range) & 8.3 & $(1.5-14)$ & 16 & $(14-18)$ & $<.001$ \\
\hline Median interval between diagnosis and HSCT, y (range) & 2.9 & $(0.5-10)$ & 3.3 & $(0.5-8.8)$ & N.S. \\
\hline \multicolumn{6}{|l|}{ Donor } \\
\hline MFD & 168 & (50) & 31 & (51) & N.S. \\
\hline UD & 166 & (50) & 30 & (49) & \\
\hline HLA typing resolution for UD & & & & & \\
\hline High resolution & 113 & (68) & 21 & (70) & N.S. \\
\hline Low resolution & 53 & (32) & 9 & (30) & \\
\hline Stem cell source & & & & & \\
\hline BM & 314 & (94) & 55 & (90) & N.S. \\
\hline PB & 20 & (6) & 6 & (10) & \\
\hline Infused cell dose & & & & & \\
\hline $\mathrm{BM}$, nucleated cells $\times 10^{8} / \mathrm{kg}$ (range) & 4.2 & $(1-11.1)$ & 3.1 & $(1.3-5.9)$ & N.S. \\
\hline $\mathrm{PB}, \mathrm{CD} 34^{+}$cells $\times 10^{6} / \mathrm{kg}$ (range) & 10.6 & $(3.3-18.7)$ & 6.1 & $(5.0-10.1)$ & N.S. \\
\hline Conditioning regimen & & & & & \\
\hline TBI & 301 & (90) & 56 & (92) & N.S. \\
\hline Chemotherapy & 31 & (9) & 5 & (8) & \\
\hline Missing/unknown & 2 & (1) & 0 & (0) & \\
\hline GvHD prophylaxis & & & & & \\
\hline MFD & & & & & \\
\hline $\mathrm{CsA} \pm \mathrm{PDN}$ & 151 & (90) & 24 & (77) & N.S. \\
\hline $\mathrm{CsA}+\mathrm{ATG}$ & 4 & (2) & 0 & (0) & \\
\hline CsA + MTX & 12 & (7) & 5 & (16) & \\
\hline $\mathrm{CsA}+\mathrm{MTX}+\mathrm{ATG}$ & 1 & (1) & 2 & (7) & \\
\hline UD & & & & & \\
\hline $\mathrm{CsA} \pm \mathrm{PDN}$ & 3 & (1) & 0 & (0) & N.S. \\
\hline CsA + ATG & 7 & (4) & 1 & (3) & \\
\hline CsA + MTX & 29 & (18) & 3 & (10) & \\
\hline $\mathrm{Cs} A+\mathrm{MTX}+\mathrm{ATG} \pm \mathrm{PDN}$ & 127 & (77) & 26 & (87) & \\
\hline
\end{tabular}

WBC indicates white blood cell; PB, peripheral blood; TBI, total body irradiation; PDN, prednisone; ATG, anti-thymocyte globulin; MTX, methotrexate; and N.S., not significant. 

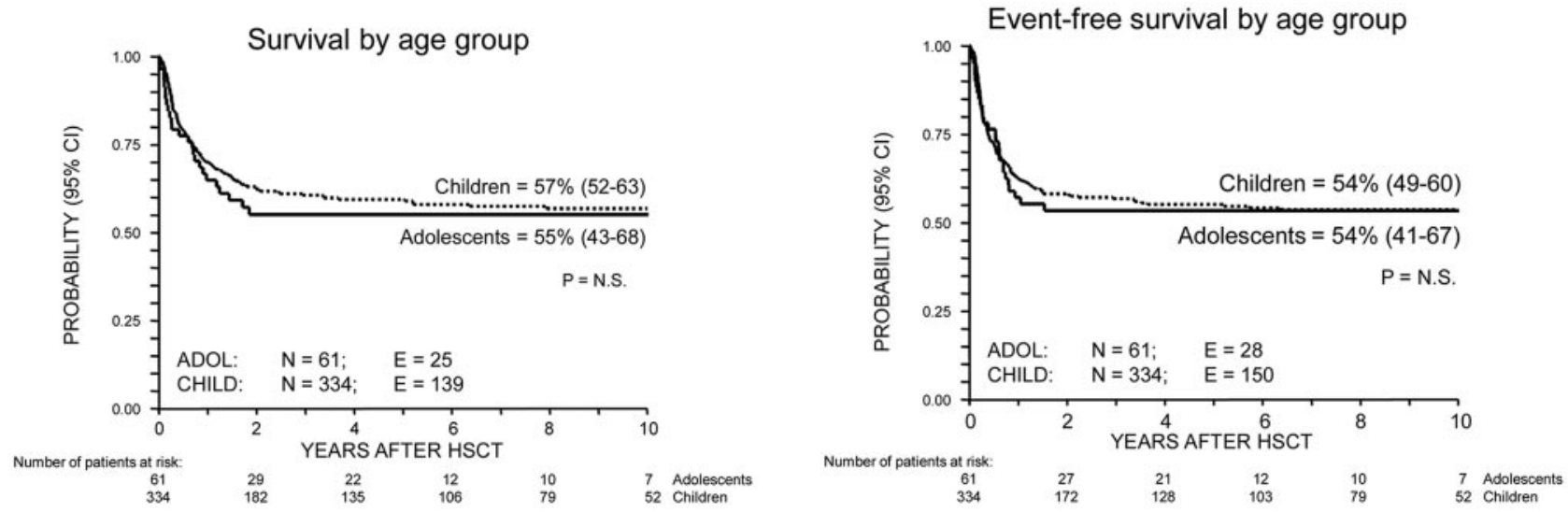

Figure 1. Cumulative incidence of TRM and relapse for ALL CR2 patients. Curves representing TRM (top) and relapse (bottom) are shown for children ( $<14$ years of age; dotted line) and adolescents ( $\geq 14$ years of age; continuous line). $P=$ N.S. N indicates number; $E$, events.

onset, whereas aGvHD preceded cGvHD in the remaining 97 patients.

\section{Transplant-related mortality}

Eighty-five patients $(22 \%)$ died of transplantation-related causes at a median time of 89 days (range, 5 days- 6 years) after the allograft. Details on causes and timing of death are reported in Table 2.

The 100-day and 10-year cumulative incidences of TRM were $12 \%$ (95\% CI, 9\%-16\%), and 22\% (95\% CI, 18\%-27\%) for children and $18 \%(95 \% \mathrm{CI}, 11 \%-31 \%)$ and $23 \%(95 \% \mathrm{CI}$, $14 \%-36 \%$ ) for adolescents, respectively, with no statistically significant difference between the 2 groups (Figure 1).

Transplants from an UD $(P<.0001)$, especially if typed with low-resolution techniques $(P<.0001)$, occurrence of grade IV aGvHD $(P<.0001)$ and of extensive cGvHD $(P<.0001)$, were significantly associated, in univariate analysis, with a higher risk of TRM (Table 3). Among patients transplanted from an UD, the cumulative incidence of TRM was significantly higher before 2000 and when low-resolution HLA typing was performed (see Table 3 for details). Multivariate analysis confirmed that recipients of transplantation from an UD typed with low-resolution techniques, patients who experienced grade IV aGvHD, and those who developed $\mathrm{cGvHD}$ had a significantly increased risk of TRM (Table 4).

\section{Relapse}

Leukemia relapse occurred in 93 patients (24\%) at a median time of 186 days after the allograft (range, 29 days-5.5 years). The cumulative incidence of relapse for children and adolescents was $25 \%$ (95\% CI, 20\%-39) and 22\% (95\% CI, 14-36), respectively $(P=$ N.S.; Figure 1), with no difference in the timing of relapse between the 2 groups. Details on the timing of disease-related deaths are reported in Table 2.

Univariate analyses demonstrated that S3 and S4 BFM classes of risk $(P<.0001)$, absence of both aGvHD $(P<.0001)$ and use of a chemotherapy-based conditioning regimen were associated with an increased risk of disease recurrence after HSCT (Table 3). Multivariate analysis confirmed that the former 2 of these 3 risk factors remained significantly correlated with an increased risk of relapse (Table 4).

\section{Overall and event-free survival}

Overall, 164 patients died at a median of 6 months after transplantation, with the values for children and adolescents being 6 months (range, 11 days-6 years) and 5 months (range, 5 days-1.8 years), respectively $(P=$ N.S.; Figure 2$)$. On the whole, 231 patients $(58 \%)$ were alive at time of data analysis and 217 of them $(55 \%$ of the study population) were disease-free. The 10-year KaplanMeier estimates of OS and EFS were 57\% (95\% CI, 52\%-62\%) and $54 \%$ (95\% CI, 49\%-59\%), respectively, for the whole cohort of patients. The 10-year EFS was 54\% (95\% CI, 49\%-60\%) for children and $54 \%(95 \% \mathrm{CI}, 41 \%-67 \%)$ for adolescents, respectively $(P=$ N.S.; Figure 2$)$.

Univariate analysis of the different patient-, donor-, and transplantation-related characteristics potentially influencing the clinical outcome showed that male sex $(P<.01)$, S3 and S4 BFM risk class at first relapse $(P<.0001)$, UD donor $(P<.02)$, and either absence or grade IV aGvHD $(P<.0001)$ significantly correlated with a lower EFS (Table 3). Because of the lower risk of TRM, patients transplanted from an UD donor after 2000 had a better probability of EFS, comparable with that of patients transplanted from an MFD in the same time period (see Table 3 for details). Likewise, patients transplanted from an UD selected using highresolution molecular typing had a probability of EFS similar to that of patients given the allograft from an MFD and significantly better than that of patients transplanted from an unrelated volunteer selected using low-resolution typing. S3 and S4 BFM class at first relapse and absence of aGvHD remained significantly associated with a worse EFS also in the multivariate Cox regression model (Table 4).

\section{Discussion}

Age has been reported to have a significant impact on survival in pediatric patients with ALL, as well as on the clinical outcome of patients receiving allogeneic HSCT. 2,4,5,22

Adolescent patients with cancer are a peculiar population, because they are at a unique point in their lives where autonomy must be balanced with guidance and support from their families and treating physicians. According to data provided by the Italian Association of Tumor Registries, each year 80 adolescents are expected to be diagnosed with ALL in Italy. ${ }^{23}$ An analysis carried out by the Epidemiology and Biostatistics Working Group of the AIEOP on patients diagnosed between 2001 and 2006 showed that only $43 \%$ of the Italian adolescents with ALL were diagnosed and treated in AIEOP institutions, with the remaining part being treated in adult centers (Roberto Rondelli, Clinica Pediatrica Università di 
Table 2. Causes and timing of death in study population

\begin{tabular}{|c|c|c|c|c|c|}
\hline & \multicolumn{2}{|c|}{$\begin{array}{l}\text { Children } \\
(n=334)\end{array}$} & \multicolumn{2}{|c|}{$\begin{array}{l}\text { Adolescents } \\
(n=61)\end{array}$} & \multirow[b]{2}{*}{$\begin{array}{c}\text { Total } \\
(\mathrm{N}=395)\end{array}$} \\
\hline & $\begin{array}{l}\text { First } \\
100 \mathrm{~d} \\
\text { after } \\
\text { HSCT }\end{array}$ & $\begin{array}{l}\text { After } \\
100 \mathrm{~d} \\
\text { from } \\
\text { HSCT }\end{array}$ & $\begin{array}{c}\text { First } \\
100 \mathrm{~d} \\
\text { after } \\
\text { HSCT }\end{array}$ & $\begin{array}{c}\text { After } \\
100 \mathrm{~d} \\
\text { from } \\
\text { HSCT }\end{array}$ & \\
\hline \multicolumn{6}{|l|}{ Cause of death } \\
\hline aGvHD & 12 & 6 & 2 & 0 & 20 \\
\hline cGvHD & 0 & 9 & 0 & 2 & 11 \\
\hline Hemorrhage & 3 & 1 & 2 & 0 & 6 \\
\hline ARDS & 3 & 1 & 0 & 0 & 4 \\
\hline Idiopathic pneumonia & 1 & 1 & 1 & 0 & 3 \\
\hline Aspergillus pneumonia & 2 & 1 & 0 & 0 & 3 \\
\hline Pneumocystis pneumonia & 0 & 0 & 1 & 0 & 1 \\
\hline HCMV pneumonia & 2 & 0 & 0 & 1 & 3 \\
\hline Bacterial infections & 6 & 6 & 2 & 0 & 14 \\
\hline Fungal infections & 2 & 0 & 0 & 0 & 2 \\
\hline Viral infections & 1 & 1 & 1 & 0 & 3 \\
\hline Veno-occlusive disease & 1 & 1 & 1 & 0 & 3 \\
\hline Multiorgan failure & 4 & 4 & 1 & 0 & 9 \\
\hline EBV-PTLD & 2 & 0 & 0 & 0 & 2 \\
\hline TTP & 1 & 0 & 0 & 0 & 1 \\
\hline Total transplant-related deaths & 40 & 31 & 11 & 3 & 85 \\
\hline Disease progression & 4 & 64 & 1 & 10 & 79 \\
\hline Total & 44 & 95 & 12 & 13 & 164 \\
\hline
\end{tabular}

ARDS indicates acute respiratory distress syndrome; HCMV, human cytomegaIovirus; EBV-PTLD, Epstein-Barr virus-related post-transplant lymphoproliferative disease; and TTP, thrombotic thrombocytopenic purpura.

Bologna, Policlinico Sant'Orsola-Bologna, personal communication, January, 2011). Thus, the adolescents may be treated by either pediatric or adult oncologists. As such, the treating physicians, including transplanters, may view the 14- to 18-year-old patient as an older child or as a younger adult.

Two questions need to be answered before defining how and where adolescents should be treated. Which is the best frontline therapy for this group of patients? Who should be responsible for HSCT?

In general, adolescents compared with children with cancer tend to have worse OS and health outcomes at the end of treatment, with this difference possibly being because of the treatment protocol used. In this particular regard, many retrospective analyses of the adolescent age group with newly diagnosed ALL treated according to either pediatric or adult protocols showed a statistically significantly superior outcome for patients treated with pediatric regimens. ${ }^{22,24,25}$ A lower relapse rate accounted for the superior results observed in adolescents treated according to pediatric protocols. Several factors may have contributed to explain this different outcome, which cannot be interpreted in view of differences in the distribution of prognostic factors between the population enrolled in pediatric and in adult trials. A major role is certainly played by differences in protocol design and treatment intensity, with pediatric protocols including more non-myelosuppressive drugs with demonstrated activity on ALL blasts, such as L-asparaginase, glucocorticoids, and vincristine. ${ }^{22,24,25}$ Moreover, central nervous system prophylaxis was administered earlier, with greater frequency and for a more prolonged period in pediatric trials. ${ }^{22,24,25} \mathrm{~A}$ minor role also may be played by a more accurate administration of therapy in pediatric institutions, because of a peculiar attitude of pediatricians concerning the need to maintain the prescribed doses and schedules, and a possibly better compliance of adolescent patients treated in a pediatric facility. ${ }^{26}$ Actually, worldwide, the outcome of adolescents with ALL has significantly improved over time. ${ }^{2,22,24-28}$ Patients enrolled in our study were treated according
Table 3. Ten-year EFS, RR, and TRM according to recipient, donor, and transplant characteristics: univariate analyses

\begin{tabular}{|c|c|c|c|}
\hline Variable & $\begin{array}{c}\text { EFS, \% } \\
(95 \% \mathrm{Cl})\end{array}$ & $\begin{array}{c}\text { RR, \% } \\
(95 \% \mathrm{Cl})\end{array}$ & $\begin{array}{l}\text { TRM, \% } \\
(95 \% \mathrm{Cl})\end{array}$ \\
\hline \multicolumn{4}{|c|}{ Age at transplant } \\
\hline Children & $54(49-60)$ & $24(20-29)$ & $22(18-27)$ \\
\hline Adolescents & $54(41-67)$ & $23(15-36)$ & $23(14-36)$ \\
\hline$P$ & N.S. & N.S. & N.S. \\
\hline \multicolumn{4}{|l|}{ Sex } \\
\hline Male & $49(42-55)$ & $26(22-33)$ & $25(20-31)$ \\
\hline Female & $65(57-73)$ & $19(13-27)$ & $16(11-24)$ \\
\hline$P$ & 0.0051 & N.S. & 0.059 \\
\hline \multicolumn{4}{|c|}{ Immunophenotype } \\
\hline B lineage & $55(49-61)$ & $23(18-28)$ & $22(18-28)$ \\
\hline $\mathrm{T}$ lineage & $47(31-63)$ & $28(16-47)$ & $25(14-44)$ \\
\hline$P$ & N.S. & N.S. & N.S. \\
\hline \multicolumn{4}{|c|}{ BFM class at relapse } \\
\hline $\mathrm{S} 1+\mathrm{S} 2$ & $63(55-70)$ & $17(12-24)$ & $20(15-27)$ \\
\hline $\mathrm{S} 3+\mathrm{S} 4$ & $38(28-47)$ & $37(29-47)$ & $26(19-35)$ \\
\hline Unknown & $58(48-68)$ & $21(15-30)$ & $21(14-31)$ \\
\hline$P$ & $<0.0001$ & 0.0002 & N.S. \\
\hline \multicolumn{4}{|c|}{ Year of HSCT, all pts } \\
\hline Before 2000 & $49(41-56)$ & $28(22-36)$ & $23(18-31)$ \\
\hline 2000 or later & $58(51-65)$ & $21(16-27)$ & $21(16-27)$ \\
\hline$P$ & 0.046 & 0.083 & N.S. \\
\hline \multicolumn{4}{|c|}{ Year of HSCT, MFD } \\
\hline Before 2000 & $55(46-64)$ & $30(23-39)$ & $15(10-23)$ \\
\hline 2000 or later & $65(53-76)$ & $20(13-31)$ & $15(8-27)$ \\
\hline$P$ & N.S. & N.S. & N.S. \\
\hline \multicolumn{4}{|c|}{ Year of HSCT, UD } \\
\hline Before 2000 & $35(22-48)$ & $24(15-39)$ & $41(30-56)$ \\
\hline 2000 or later & $55(46-63)$ & $21(15-30)$ & $24(18-32)$ \\
\hline$P$ & 0.0072 & N.S. & $<0.0001$ \\
\hline \multicolumn{4}{|l|}{ Donor } \\
\hline MFD & $59(52-66)$ & $26(20-33)$ & $15(11-21)$ \\
\hline UD & $50(42-57)$ & $22(17-29)$ & $29(23-36)$ \\
\hline$P$ & 0.022 & N.S. & 0.0006 \\
\hline \multicolumn{4}{|c|}{ HLA typing resolution } \\
\hline MFD & $59(52-66)$ & $26(20-33)$ & $15(11-21)$ \\
\hline HR UD & $57(48-65)$ & $21(15-29)$ & $22(16-31)$ \\
\hline LR UD & $35(23-47)$ & $23(14-36)$ & $42(31-56)$ \\
\hline$P$ & 0.0003 & N.S. & $<0.0001$ \\
\hline \multicolumn{4}{|c|}{ Conditioning regimen } \\
\hline $\mathrm{TBI}+\mathrm{CHT}$ & $56(51-61)$ & $23(19-27)$ & $22(18-26)$ \\
\hline CHT alone & $44(27-60)$ & $31(19-50)$ & $26(15-45)$ \\
\hline$P$ & N.S. & 0.011 & N.S. \\
\hline \multicolumn{4}{|c|}{ Infused nucleated cells } \\
\hline$<4 \times 10^{8} / \mathrm{kg}$ & $57(49-64)$ & $22(17-29)$ & $21(16-28)$ \\
\hline$\geq 4 \times 10^{8} / \mathrm{Kg}$ & $57(49-64)$ & $26(20-33)$ & $18(13-25)$ \\
\hline$P$ & N.S. & N.S. & 0.0009 \\
\hline \multicolumn{4}{|l|}{ aGvHD grade } \\
\hline 0 & $15(1-28)$ & $67(51-87)$ & $19(8-41)$ \\
\hline I & $66(57-75)$ & $23(16-32)$ & $11(7-19)$ \\
\hline II & 64 (56-73) & $20(14-29)$ & $15(10-23)$ \\
\hline III & $58(40-75)$ & $16(7-36)$ & $27(15-47)$ \\
\hline IV & $14(1-27)$ & $4(1-24)$ & $82(69-98)$ \\
\hline$P$ & $<0.0001$ & $<0.0001$ & $<0.0001$ \\
\hline \multicolumn{4}{|l|}{ cGvHD } \\
\hline Absent & $66(59-72)$ & $28(23-35)$ & $6(4-11)$ \\
\hline Limited & $76(66-87)$ & $12(6-23)$ & $11(6-23)$ \\
\hline Extensive & $57(41-73)$ & $22(12-40)$ & $21(11-39)$ \\
\hline$P$ & N.S. & 0.054 & $<0.0001$ \\
\hline
\end{tabular}

The log-rank test was used for comparisons of EFS probabilities, whereas the Gray test was used to compare cumulative incidences of relapse and TRM.

HR indicates high-resolution HLA typing (4 digits) for both first- and second-class HLA antigens; LR, low-resolution HLA typing (2 digits) for first-class HLA antigens and high-resolution HLA typing (4 digits) for second-class HLA antigens; CHT, chemotherapy; and N.S., not significant. 
Table 4. Multivariate analysis of variables influencing EFS, RR, and 12-month TRM overall

\begin{tabular}{|c|c|c|c|}
\hline & $\mathbf{R R}$ & $95 \% \mathrm{Cl}$ & $P$ \\
\hline \multicolumn{4}{|l|}{ EFS: risk of treatment failure } \\
\hline \multicolumn{4}{|l|}{ Age at transplant } \\
\hline Adolescents vs children & 1.005 & $0.57-1.76$ & N.S. \\
\hline \multicolumn{4}{|l|}{ Sex } \\
\hline Male vs female & 0.91 & $0.61-1.36$ & N.S. \\
\hline \multicolumn{4}{|l|}{ BFM class at relapse } \\
\hline $\mathrm{S} 3+\mathrm{S} 4 \mathrm{vs} \mathrm{S} 1+\mathrm{S} 2$ & 2.50 & $1.72-3.64$ & $<.0001$ \\
\hline \multicolumn{4}{|l|}{ HLA typing resolution } \\
\hline HR vs MFD & 1.47 & $0.84-2.59$ & N.S. \\
\hline LR vs MFD & 1.24 & $0.78-2.00$ & N.S. \\
\hline \multicolumn{4}{|l|}{ Conditioning regimen } \\
\hline TBI vs chemotherapy & 2.36 & $0.94-5.88$ & .066 \\
\hline \multicolumn{4}{|l|}{ aGvHD } \\
\hline Grade I vs 0 & 0.31 & $0.17-0.56$ & .0001 \\
\hline Grade II vs 0 & 0.27 & $0.15-0.50$ & $<0.0001$ \\
\hline Grade III vs 0 & 0.46 & $0.22-0.99$ & .047 \\
\hline Grade IV vs 0 & 1.24 & $0.64-2.41$ & N.S. \\
\hline \multicolumn{4}{|l|}{ Year of HSCT } \\
\hline 2000 or later vs before 2000 & 0.63 & $0.36-1.08$ & N.S. \\
\hline \multicolumn{4}{|l|}{ Relapse } \\
\hline \multicolumn{4}{|l|}{ Age at transplant } \\
\hline Adolescents vs children & 1.001 & $0.56-1.80$ & N.S. \\
\hline \multicolumn{4}{|l|}{ Sex } \\
\hline Male vs female & 1.33 & $0.83-2.15$ & N.S. \\
\hline \multicolumn{4}{|l|}{ BFM class at relapse } \\
\hline $\mathrm{S} 3+\mathrm{S} 4 \mathrm{vs} \mathrm{S} 1+\mathrm{S} 2$ & 2.50 & $1.58-3.95$ & .0001 \\
\hline \multicolumn{4}{|l|}{ Conditioning regimen } \\
\hline TBI vs chemotherapy & 1.03 & $0.56-1.91$ & N.S. \\
\hline \multicolumn{4}{|l|}{ aGvHD } \\
\hline Grade I vs 0 & 0.48 & $0.28-0.82$ & .008 \\
\hline Grade II vs 0 & 0.45 & $0.25-0.81$ & .008 \\
\hline Grade III vs 0 & 0.32 & $0.12-0.83$ & .019 \\
\hline Grade IV vs 0 & 0.06 & $0.01-0.47$ & .008 \\
\hline \multicolumn{4}{|l|}{ cGvHD } \\
\hline Limited vs absent & 0.44 & $0.21-0.91$ & .026 \\
\hline Extensive vs absent & 0.74 & $0.33-1.64$ & N.S. \\
\hline \multicolumn{4}{|l|}{ Year of HSCT } \\
\hline 2000 or later vs before 2000 & 0.60 & $0.44-1.53$ & N.S. \\
\hline \multicolumn{4}{|l|}{ TRM } \\
\hline Age at transplant & & & \\
\hline Adolescents vs children & 1.61 & $0.87-2.96$ & N.S. \\
\hline Sex & & & \\
\hline Male vs female & 1.23 & $0.74-2.05$ & N.S. \\
\hline BFM class at relapse & & & \\
\hline $\mathrm{S} 3+\mathrm{S} 4 \mathrm{vs} \mathrm{S} 1+\mathrm{S} 2$ & 1.27 & $0.81-1.97$ & N.S. \\
\hline HLA typing resolution & & & \\
\hline HR vs MFD & 1.55 & $0.87-2.76$ & N.S. \\
\hline LR vs MFD & 3.04 & $1.74-5.29$ & .0001 \\
\hline Conditioning regimen & & & \\
\hline TBI vs chemotherapy & 0.73 & $0.37-1.47$ & N.S. \\
\hline aGvHD & & & \\
\hline Grade I vs 0 & 0.49 & $0.24-0.99$ & .046 \\
\hline Grade II vs 0 & 0.82 & $0.43-1.54$ & N.S. \\
\hline Grade III vs 0 & 1.50 & $0.63-3.55$ & N.S. \\
\hline Grade IV vs 0 & 6.81 & $3.74-12.39$ & $<.0001$ \\
\hline cGvHD & & & \\
\hline Limited vs absent & 0.32 & $0.14-0.76$ & .0095 \\
\hline Extensive vs absent & 0.66 & $0.32-1.37$ & N.S. \\
\hline Year of HSCT & & & \\
\hline 2000 or later vs before 2000 & 0.99 & $0.59-1.67$ & N.S. \\
\hline
\end{tabular}

N.S. indicates not significant.

to frontline protocols of several studies, namely, AIEOP ALL 82, $87,88,91,95$, and 2000, which showed a continuous improvement in final outcome. Indeed, the 15-year EFS increased from $52.5 \%$ for the 902 eligible patients included in the study 82 to $67.7 \%$ for the 1192 children enrolled in study $91 .^{2}$ The 10-year EFS of in the 1743 eligible patients enrolled in study 95 was $71.7 \%,{ }^{2}$ whereas, for what concerns patients treated according to the study AIEOPBFM 2000, those with B-cell precursor (4016 children) and T-lineage ALL (464 children) had a 7-year EFS of $80.4 \%$ and $75.9 \%$, respectively. ${ }^{28,29}$ The improved outcome of patients treated with frontline chemotherapy in Italy was documented in both children and adolescents (data not shown).

Concerning transplantation, previously published studies reported that in pediatric patients older age also has a relevant impact on the probability of EFS after HSCT., ${ }^{4,30}$ Indeed, Bunin et al reported that patients more than 15 years of age undergoing UD HSCT for ALL had a worse outcome than younger patients, with TRM being $60 \%$ in the former compared with $38 \%$ in the latter patients. ${ }^{4}$ For a correct and meaningful interpretation of these results, it has to be highlighted that the 363 patients analyzed in that study were treated in several institutions without homogeneous risk criteria at diagnosis, with different both frontline and post-relapse therapy protocols, and varying conditioning regimens.

A study by Woolfrey et al on UD HSCT for childhood ALL showed that both advanced disease phase $(P<.0001)$ and age more than 10 years $(P<.002)$ were predictors of worse outcome. ${ }^{5}$ In multivariate analyses, older age correlated with greater risk of aGvHD and organ toxicity, with these conditions lead to an increased TRM. More recently, a retrospective survey of the Paediatric Diseases Working Party of the European Group for Blood and Marrow Transplantation on outcome after HSCT in pediatric patients over the last 3 decades showed that age more than 14 years significantly correlated with higher TRM, both in univariate and multivariate analysis. ${ }^{30}$

In contrast with all these data, our study provides evidence that the outcome after HSCT for ALL in CR2 is similar in children and adolescents when both are treated in the same pediatric institutions, in the same period.

The strength of our analysis lies in the even distribution of initial clinical and hematologic features between children and adolescents and in the uniformity of the anti-leukemia frontline therapy administered according to the current AIEOP-BFM protocols. ${ }^{2,28,29}$ A homogeneous approach in both children and adolescents also was used in the treatment of relapse. Also the conditioning regimens used by the 11 centers participating in the study were substantially homogeneous, without significant differences among them.

GVHD is known to be an important factor influencing TRM and EFS. It has been reported that among patients transplanted from an HLA-identical sibling and given GVHD prophylaxis consisting of a combination of methotrexate and CsA, older recipient age $(P<.0001)$ was the single most important risk factor for cGvHD. ${ }^{31}$ More recently, a paper published by the AIEOP-HSCT group showed that recipient age more than 15 years significantly correlated with a higher risk of cGvHD, but ultimately, however, did not influence the probability of EFS. ${ }^{32}$ In our study, the incidence and severity of both aGvHD and cGvHD was similar in children and adolescents, and this observation can contribute to explain the comparable risk of death from transplantation-related causes that we observed in the 2 groups. Nevertheless, both aGVHD and cGVHD were responsible for most transplantation-related deaths in children and in adolescents, and in multivariate analysis, aGvHD and cGvHD were risk factors with a detrimental impact on TRM.

Notably, in our cohort, the risk of disease recurrence after HSCT also did not differ between children and adolescents. 

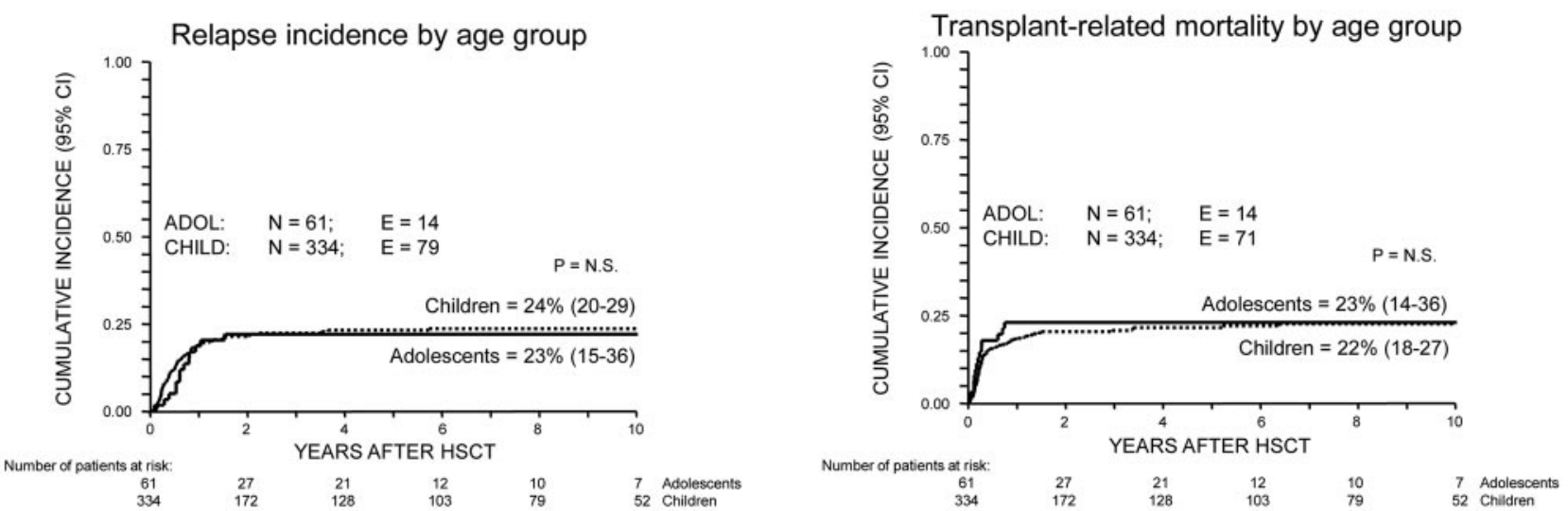

Figure 2. Probability of overall and of event-free survival for ALL CR2 patients. Curves representing survival (top) and disease-free survival (bottom) are shown for children ( $<14$ years of age; dotted line) and adolescents ( $\geq 14$ years of age; continuous line). $P=$ N.S.

Concerning other variables potentially influencing leukemia relapse after HSCT, we confirmed that BFM stratification in different classes of risk is valuable in predicting disease recurrence after transplantation, as reported in a previously published analysis by our group. ${ }^{33}$ The role of graft-versus-leukemia (GvL) in ALL is still debated, with some studies reporting either no effect or a limited effect restricted to aGvHD only. ${ }^{34}$ By contrast, other analyses have noted a correlation of GvL with acute or cGvHD. ${ }^{32,35}$ In our study, the occurrence of both aGvHD and cGvHD decreased the risk of leukemia relapse, and patients who did not experience aGVHD had an increased risk of treatment failure compared with those who developed grade I-III acute GVHD. Altogether, these data provide support to the concept that a GVHD-related GvL effect is important for preventing disease recurrence in childhood ALL.

The final outcome of our patients transplanted from an unrelated volunteer did not differ in multivariate analysis from that of patients given HSCT from an HLA-identical sibling and the 10 -year EFS of $50 \%$ observed in our cohort of unrelated HSCT recipients compares favorably with results reported in the studies by Bunin et $\mathrm{al}^{4}$ and Woolfrey et $\mathrm{al}^{5}{ }^{5}$ Indeed, we observed that for patients transplanted after 2000 from an UD the risk of treatmentrelated death was significantly lower than that of patients transplanted before that date, this resulting into a better probability of EFS. We have previously provided evidence that outcome of children with CR2 ALL given HSCT from an UD has improved over time, and the present results confirm that currently, thanks to the improvements HLA typing obtained through the use of high-resolution molecular techniques and the optimization of GVHD prevention and treatment, post-transplantation outcome is not influenced by the type of donor used, either related or unrelated. ${ }^{33}$ Support to this interpretation is provided by the observation that the use of high-resolution molecular typing for both HLA class I and II loci resulted into a significantly lower TRM and better EFS compared with the use of low-resolution typing (see Table 3 for details).

In conclusion, this study shows that adolescents with CR2 ALL given HSCT from a matched sibling or an UD, performed in pediatric centers, have an outcome that does not differ from that of patients younger than 14 years of age. Thus, in view of these data, patient age has not to be considered any longer a risk factor when considering or discussing the risks and benefits of the transplant option for pediatric patients with CR2 ALL. Information about prognostic factors provided by the current study also can be used to direct future strategies to further improve outcome for children with CR2 ALL who undergo allogeneic HSCT.

\section{Acknowledgments}

This study was partly supported by Associazione Italiana Ricerca sul Cancro grant "5x1000" (F.L.); a grant from Ricerca Corrente, Ospedale Bambino Gesù, Rome (F.L.); and Ricerca Finalizzata Ministeriale grant RF-IGG-2007-650460 (G.D.).

\section{Authorship}

Contribution: G.D. and F.L. designed research; G.D., M.Z., A.B., C.M., R.M., F.F., C.F., M.R., F.P., E.B., M.R., A.P.I., A.P., and F.L. treated patients; M.Z. and C.R. performed statistical analysis; all authors collected clinical data; G.D., A.B., M.Z., and F.L. analyzed and interpreted data; and G.D. and F.L. wrote the manuscript, which was revised and approved by all coauthors.

Conflict-of-interest disclosure: The authors declare no competing financial interests.

A complete list of the members of the Associazione Italiana Ematologia ed Oncologia Pediatrica Hemopoietic Stem Cell Transplant Group appears in the supplemental Appendix (available on the Blood Web site; see the Supplemental Materials link at the top of the online article).

Correspondence: Giorgio Dini, Dipartimento Ematologia e Oncologia Pediatrica, IRCCS G. Gaslini, Largo G. Gaslini 5, 16147 Genova, Italy; e-mail: giorgiodini@ ospedale-gaslini.ge.it.

\section{References}

1. Pui $\mathrm{CH}$, Robinson LL, Look AT. Acute lymphoblastic leukaemia. Lancet. 2008;371(9617):1030-1043.

2. Conter V, Aricò M, Basso G, et al. Long-term results of the Italian Association of Pediatric Hematology and Oncology (AIEOP) Studies 82, 87, 88, 91 and 95 for childhood acute lymphoblastic leukemia. Leukemia. 2010;24(2):255-264.
3. Gaynon PS. Childhood acute lymphoblastic leukaemia relapse. Br J Haematol. 2005;131(5):579587.

4. Bunin N, Carston M, Wall D, et al. Unrelated marrow transplantation for children with acute lymphoblastic leukemia in second remission. Blood. 2002;99(9):3151-3157.
5. Woolfrey AE, Anasetti C, Storer B, et al. Factors associated with outcome after unrelated marrow transplantation for treatment of acute lymphoblastic leukemia in children. Blood. 2002;99(6): 2002-2008.

6. Zecca M, Pession A, Messina C, et al. Total body irradiation, thiotepa, and cyclophosphamide as a 
conditioning regimen for children with acute lymphoblastic leukemia in first or second remission undergoing bone marrow transplantation with HLA-identical siblings. J Clin Oncol. 1999;17(6):1838-1846.

7. Glucksberg $\mathrm{H}$, Storb R, Fefer A, et al. Clinical manifestations of graft-versus host disease in human recipients of marrow from HLA-matched sibling donors. Transplantation. 1974;18(4):295-304.

8. Storb R, Prentice RL, Sullivan KM, et al. Predictive factors in chronic graft-versus-host disease in patients with aplastic anemia treated by bone marrow transplantation from using HLA-identical sibling donors. Ann Intern Med. 1983;98(4):461466.

9. Dallorso S, Rondelli R, Messina C, et al. Clinical benefits of granulocyte colony-stimulating factor therapy after hematopoietic stem cell transplant in children: results of a prospective randomized trial. Haematologica. 2002;87(12):1274-1280.

10. Locatelli F, Percivalle E, Comoli P, et al. Human cytomegalovirus (HCMV) infection in paediatric patients given allogeneic bone marrow transplantation: role of early antiviral treatment for $\mathrm{HCMV}$ antigenaemia on patients' outcome. Br J Haematol. 1994;88(1):64-71.

11. Henze G, Fengler R, Hartmann R, et al. Six-year experience with a comprehensive approach to the treatment of recurrent childhood acute lymphoblastic leukemia (ALL-REZ BFM 85). A relapse study of the BFM group. Blood. 1991;78(5): 1166-1172.

12. Clift R, Goldman J, Gratwohl A, Horowitz M. Proposals for standardized reporting of results of bone marrow transplantation for leukaemia. Bone Marrow Transplant. 1989;4(4):445-448.

13. Klein JP, Rizzo JD, Zhang MJ, Keiding N. Statistical methods for the analysis and presentation of the results of bone marrow transplants. Part I: unadjusted analysis. Bone Marrow Transplant. 2001;28(10):909-915.

14. Kaplan EL, Meier P. Non parametric estimation from incomplete observations. J Am Stat Assoc. 1958;53(282):457-481.

15. Gooley TA, Leisenring W, Crowley J, Storer BE. Estimation of failure probabilities in the presence of competing risks: new representations of old estimators. Stat Med. 1999;18(6):695-706.

16. Pepe MS, Longton G, Pettinger M, Mori M, Fisher LD, Storb R. Summarizing data on sur- vival, relapse, and chronic graft-versus-host disease after bone marrow transplantation: motivation for and description of new methods. $\mathrm{Br} \mathrm{J}$ Haematol. 1993;83(4):602-607.

17. Gray RJ. A class of K-sample tests for comparing the cumulative incidence of a competing risk. Ann Statist. 1988;16(3):1141-1154.

18. Cox DR. Regression models and life tables. $J$ R Stat Soc B. 1972;34(2):187-220.

19. Fine JP, Gray RJ. A proportional hazards model for the subdistribution of a competing risk. J Am Stat Assoc. 1999;94:496-509.

20. Scrucca L, Santucci A, Aversa F. Competing risk analysis using $\mathrm{R}$ : an easy guide for clinicians. Bone Marrow Transplant. 2007;40(4):381-387.

21. Scrucca L, Santucci A, Aversa F. Regression modeling of competing risk using $\mathrm{R}$ : an in depth guide for clinicians. Bone Marrow Transplant. 2010;45(9):1388-1395.

22. Boissel N, Auclerc MF, Lheritier V, et al. Should adolescents with acute lymphoblastic leukemia be treated as old children or young adults? Comparison of the French FRALLE-93 and LALA-94 trials. J Clin Oncol. 2003;21(5):774-780.

23. AIRTUM Working Group. Italian cancer figuresreport 2008. 1. Childhood cancer. Epidemiol Prev. 2008;32(2 suppl 2):1,5-13,16-35.

24. de Bont JM, Holt B, Dekker AW, van der Does-van den Berg A, Sonneveld P, Pieters R. Significant difference in outcome for adolescents with acute lymphoblastic leukaemia treated on paediatric vs adult protocols in the Netherlands. Leukemia. 2004;18(12):2032-2035.

25. Ramanujachar R, Richards S, Hann I, et al. Adolescents with acute lymphoblastic leukaemia: outcome on UK national paediatric (ALL 97) and adult (UKALL XII/E2993) trials. Pediatric Blood Cancer. 2007;48(3):254-261.

26. Hallböök H, Gustaffson G, Smedmyr B, et al. Treatment outcome in young adults and children $>10$ years of age with acute lymphoblastic leukemia in Sweden: a comparison between a pediatric protocol and an adult protocol. Cancer 2006;107(7):1551-1561.

27. Stock W, La M, Sanford B, et al. What determines the outcomes for adolescents and young adult with acute lymphoblastic leukemia treated on cooperative group protocols? A comparison of Children's Cancer Group and Cancer and Leukaemia Group B studies. Blood 2008:112(5):1646-54.
28. Conter V, Bartram CR, Valsecchi MG, et al. Molecular response to treatment redefines all prognostic factors in children and adolescents with B-cell precursor acute lymphoblastic leukemia: results in 3184 patients of the AIEOP-BFM ALL 2000 study. Blood. 2010;115(16):3206-3214.

29. Schrappe M, Valsecchi MG, Bartram CR, et al. Late MRD response determines relapse risk overall and in subsets of childhood T-cell ALL: results of the AIEOP-BFM-ALL 2000 study. Blood. 2011;118(8):2077-2084.

30. Miano M, Labopin M, Hartmann O, et al. Paediatric Diseases Working Party of the European Group for Blood and Marrow Transplantation. Haematopoietic stem cell transplantation trends in children over the last three decades: a survey by the paediatric diseases working party of the European Group for Blood and Marrow Transplantation. Bone Marrow Transplant. 2007;39(2): 89-99.

31. Carlens S, Ringden O, Remberger M, et al. Risk factors for chronic graft-versus-host disease after bone marrow transplantation: a retrospective single centre analysis. Bone Marrow Transplant. 1998;22(8):755-761.

32. Zecca M, Prete A, Rondelli R, et al. Chronic graftversus-host disease in children: incidence, risk factors, and impact on outcome. Blood. 2002; 100(4):1192-1200.

33. Locatelli F, Zecca M, Messina C, et al. Improvement over time in outcome for children with acute lymphoblastic leukemia in second remission given hematopoietic stem cell transplantation from unrelated donors. Leukemia. 2002;16(11): 2228-2237.

34. Weisdorf DJ, Nesbit ME, Ramsay NK, et al. Allogeneic bone marrow transplantation for acute lymphoblastic leukemia in remission: prolonged survival associated with acute graft-versus-host disease. J Clin Oncol. 1987;5(9):1348-1355

35. Muñoz A, Diaz-Heredia C, Diaz MA, et al. Allogeneic hemopoietic stem cell transplantation for childhood acute lymphoblastic leukemia in second complete remission-similar outcomes after matched related and unrelated donor transplant: a study of the Spanish Working Party for Blood and Marrow Transplantation in Children (Getmon). Pediatric Hematol Oncol. 2008;25(4): 245-259. 